\title{
A Study on the Ichneumonid Wasps (Hymenoptera: Ichneumonidae) from Isfahan Province, Iran
}

\author{
H. GHAHARI ${ }^{1 *}$ and N. S. GADALLAH ${ }^{2}$ \\ ${ }^{1}$ Department of Plant Protection, Yadegar - e-Imam Khomeini (RAH) Branch, \\ Islamic Azad University, Tehran, Iran \\ ${ }^{2}$ Entomology Department, Faculty of Science, Cairo University, Giza, Egypt
}

(Received: 6 April 2015; accepted: 4 June 2015)

\begin{abstract}
The fauna of ichneumonid wasps (Hymenoptera: Ichneumonidae) from Isfahan province (Iran) is studied in this paper. In total 28 species from 24 genera and 6 subfamilies (Campopleginae, Cryptinae, Ichneumoninae, Ophioninae, Pimplinae and Tryphoninae) were collected and identified. Encrateola laevigata (Ratzeburg, 1848), Mesoleptus laticintus (Walker, 1874) (Cryptinae), Hepiopelmus melanogaster (Gmelin, 1790) (Ichneumonidae), and Apechthis quadridentata (Thomson, 1877) (Pimplinae) are new records for the fauna of Iran.
\end{abstract}

Keywords: Hymenoptera, Ichneumonidae, fauna, Isfahan, Iran.

Ichneumonidae (Hymenoptera) is the biggest hymenopteran family with 51 generally recognized subfamilies, 1579 genera and 24,281 described species, with an estimated 60,000 extant species (Townes, 1969; Yu et al., 2012; Çoruh et al., 2014). Ichneumonidae is represented with about 8711 species in the Palaearctic region (Yu et al., 1997). Ichneumonids are parasitoids of immature holometabolous insects such as Coleoptera, Diptera, Hymenoptera, Lepidoptera, Rhaphidioptera, Trichoptera and also other arthropods such as Chelicerata, adult Araneae and Pseudoscorpionida (Townes, 1969). The number of known species of Ichneumonidae increases rapidly in the world (Çoruh et al., 2014).

Due to the great diversity and difficulties in identification of many species in this group, our knowledge of the Iranian fauna remains insufficient. The Ichneumonidae fauna of Iran is not well studied. In the checklist from Barahoei et al. (2012) 502 species belonging to 189 genera and 24 subfamilies are listed from Iran. Later, many authors (Barahoei et al., 2014a, b, 2015; Ghahari and Jussila, 2014a, b, 2015) have made contributions to the Iranian fauna. The most important faunistic work on the ichneumonids of Isfahan province was conducted by Barahoei et al. (2015), who reported 18 species from 16 genera and 7 subfamilies. Additionally Barahoei et al. (2014b) reported 9 species from 9 genera of the subfamily Cryptinae from Isfahan province.

\footnotetext{
* Corresponding author; e-mail: hghahari@yahoo.com
} 
Isfahan province $\left(32.6577^{\circ} \mathrm{N} 51.6692^{\circ} \mathrm{E}\right)$ is located in the center of the country and covers an area of approximately $107,027 \mathrm{~km}^{2}$. The province has a moderate and dry climate on the whole, ranging between $40.6{ }^{\circ} \mathrm{C}$ and $10.6{ }^{\circ} \mathrm{C}$ on a cold day in the winter season. The average annual temperature has been recorded as $16.7^{\circ} \mathrm{C}$ and the annual rainfall on an average has been reported as $116.9 \mathrm{~mm}$. The aim of this paper is to increase the knowledge of the ichneumonid fauna in Isfahan province.

\section{Materials and Methods}

Through the samplings by Malaise trap and sweeping net for collecting the ichneumonid wasps in different regions of Isfahan province several specimens were collected. The sampled regions in this research are Anarak, Aran-Bidgol, Ardestan, Fereydun-Shahr, Golpaygan, Isfahan, Kashan, Khansar, Khomeyni-Shahr, Nain, Najaf-Abad, Semirom, Shahreza. Classification and nomenclature of Ichneumonidae suggested by Yu et al. (2012) have been followed.

\section{Results}

In a total 28 ichneumonid species from 24 genera and subgenera and 6 subfamilies were collected from different regions of Isfahan province. The list of species is given below alphabetically.

\section{Subfamily Campopleginae \\ Genus Diadegma Förster, 1869}

\section{Diadegma fenestrale (Holmgren, 1860)}

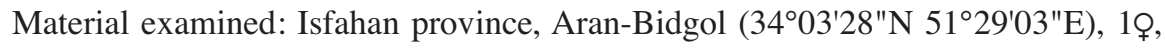
10', April 2012.

Distribution outside Iran: Afghanistan, Austria, Azerbaijan, Belgium, Bulgaria, Croatia, Cyprus, Czech Republic, Denmark, Finland, France, Germany, Hungary, Iceland, India, Ireland, Israel, Italy, Japan, Kazakhstan, Latvia, Lithuania, Moldova, Morocco, the Netherlands, New Zealand, Norway, Pakistan, Philippines, Poland, Portugal, Romania, Russia, Spain, Sri Lanka, Sweden, Switzerland, Turkey, Turkmenistan, Ukraine, UK, former Yugoslavia (Yu et al., 2012).

\section{Genus Lemophagus Townes, 1965}

\section{Lemophagus curtus Townes, 1965}

Material examined: Isfahan province, Anarak (3318'40"N 5341'54"E), 1Q, April 2006.

Distribution outside Iran: Austria, Bulgaria, Czech Republic, Denmark, France, Germany, Italy, Poland, Romania, Russia, Sweden, Switzerland, USA, Ukraine, UK (Yu et al., 2012). 


\section{Subfamily Cryptinae \\ Genus Aclastus Förster, 1869}

\section{Aclastus gracilis (Thomson, 1884)}

Material examined: Isfahan province, Fereydun-Shahr (3256'28"N 5007'16"E), 1 , June 2006.

Distribution outside Iran: Austria, Azerbaijan, Belgium, Bulgaria, Canary Islands, former Czechoslovakia, Denmark, Faeroe Islands, Finland, France, Germany, Greenland, Hungary, Iceland, Ireland, Italy, Madeira Islands, the Netherlands, Norway, Poland, Russia, Spain, Sweden, Switzerland, Turkey, UK (Yu et al., 2012).

\section{Genus Agrothereutes Förster, 1850}

\section{Agrothereutes parvulus (Habermehl, 1926)}

Material examined: Isfahan province, Aran-Bidgol (3403'28"N 51 $\left.29^{\prime} 03^{\prime \prime} \mathrm{E}\right), 20^{\prime \prime}$, April 2012.

Distribution outside Iran: Bulgaria, France, Poland, Spain, Turkey (Yu et al., 2012).

\section{Genus Cryptus Fabricius, 1804}

\section{Cryptus minator Gravenhorst, 1829}

Material examined: Isfahan province, Golpaygan $\left(33^{\circ} 27^{\prime} 13^{\prime \prime} \mathrm{N} 50^{\circ} 17^{\prime} 18^{\prime \prime} \mathrm{E}\right), 1$, September 2005.

Distribution outside Iran: Austria, Bulgaria, former Czechoslovakia, Denmark, Finland, France, Germany, Hungary, Ireland, Italy, Latvia, Lithuania, Norway, Poland, Romania, Spain, Sweden, Turkey, UK (Yu et al., 2012).

\section{Genus Encrateola Strand, 1916}

\section{Encrateola laevigata (Ratzeburg, 1848)}

Material examined: Isfahan province, Isfahan $\left(32^{\circ} 38^{\prime} \mathrm{N} 51^{\circ} 39 ' \mathrm{E}\right), 10^{\circ}$, July 2006. New record for the fauna of Iran.

Distribution outside Iran: Afghanistan, Austria, Bulgaria, Canada, Czech Republic, Finland, France, Germany, Hungary, Ireland, Lithuania, Moldova, the Netherlands, Norway, Poland, Romania, South Africa, Sweden, Turkey, USA, Ukraine, UK (Yu et al., 2012).

\section{Genus Gelis Thunberg, 1827}

\section{Gelis agilis (Fabricius, 1775)}

Material examined: Isfahan province, Khansar (33¹3'14"N 50¹8'54"E), 2Q, April

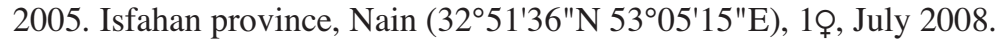

Distribution outside Iran: Albania, Austria, Azerbaijan, Belarus, Belgium, Bulgaria, Croatia, Czech Republic, Denmark, Finland, France, Germany, Greece, Hungary, Iceland, 
Ireland, Italy, Latvia, Lithuania, Moldova, the Netherlands, Norway, Poland, Romania, Russia, Spain, Sweden, Switzerland, Turkey, Ukraine, UK (Yu et al., 2012).

\section{Gelis areator (Panzer, 1804)}

Material examined: Isfahan province, Ardestan ( $\left.33^{\circ} 22^{\prime} 34^{\prime \prime N} 52^{\circ} 22^{\prime} 10^{\prime \prime E}\right), 1$, June

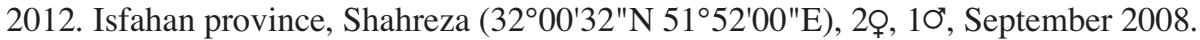

Distribution outside Iran: Austria, Azerbaijan, Belarus, Belgium, Bulgaria, China, Czech Republic, Denmark, Finland, France, Germany, Greece, Hungary, Ireland, Italy, Italy-Sicily, Japan, Korea, Latvia, Lithuania, Macedonia, Moldova, the Netherlands, New Zealand, Norway, Poland, Romania, Russia, Serbia and Montenegro, Slovakia, South Africa, Spain, Sweden, Switzerland, Ukraine, UK, former Yugoslavia (Yu et al., 2012).

\section{Genus Hoplocryptus Thomson, 1873}

\section{Hoplocryptus femoralis (Gravenhorst, 1829)}

Material examined: Isfahan province, Semirom ( $\left.31^{\circ} 24^{\prime} 51^{\prime \prime N} 51^{\circ} 34^{\prime} 10^{\prime \prime E}\right), 1$, $10^{\prime \prime}$, August 2008.

Distribution outside Iran: Algeria, Bulgaria, Croatia, Egypt, France, Germany, Greece, Israel, Italy, Italy-Sardinia, Italy-Sicily, Kazakhstan, Kyrgyzstan, Macedonia, Norway, Poland, Romania, Slovenia, Spain, Sweden, Tajikistan, Tunisia, Turkey, former Yugoslavia (Yu et al., 2012).

\section{Genus Ischnus Gravenhorst, 1829}

\section{Ischnus agitator (Olivier, 1792)}

Material examined: Isfahan province, Kashan $\left(33^{\circ} 59^{\prime} 20^{\prime \prime} \mathrm{N} 51^{\circ} 28^{\prime} 38^{\prime \prime} \mathrm{E}\right), 1$, $\mathrm{Au}-$ gust 2007.

Distribution outside Iran: Austria, Azerbaijan, Bulgaria, Croatia, former Czechoslovakia, Denmark, France, Germany, Greece, Hungary, Ireland, Italy, Italy-Sardinia, Italy-Sicily, Latvia, Poland, Romania, Spain, Sweden, Tunisia, Turkey, UK (Yu et al., 2012).

\section{Genus Meringopus Förster, 1869}

\section{Meringopus titillator (Linnaeus, 1758)}

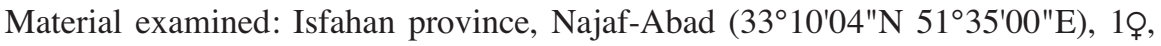
20', August 2013.

Distribution outside Iran: Afghanistan, Algeria, Armenia, Austria, Azerbaijan, Bulgaria, China, Croatia, Cyprus, Czech Republic, Denmark, Egypt, Finland, France, Germany, Greece, Hungary, Ireland, Isle of Man, Israel, Italy, Italy-Sardinia, Italy-Sicily, Jordan, Kyrgyzstan, Latvia, Morocco, the Netherlands, Norway, Palestine, Poland, Romania, Russia, Serbia and Montenegro, Spain, Sweden, Switzerland, Syria, Tajikistan, Tunisia, Turkey, Turkmenistan, Ukraine, UK, Uzbekistan, former Yugoslavia (Yu et al., 2012). 


\section{Genus Mesoleptus Gravenhorst, 1829}

Mesoleptus laticinctus (Walker, 1874)

Material examined: Isfahan province, Nain $\left(32^{\circ} 51^{\prime} 36^{\prime \prime} \mathrm{N} 53^{\circ} 05^{\prime} 15^{\prime \prime E}\right), 10^{\prime \prime}$, July 2008. New record for the fauna of Iran.

Distribution outside Iran: Austria, Azerbaijan, Belgium, Bulgaria, China, Czech Republic, Denmark, Estonia, Finland, France, Germany, Greece, Hungary, Italy, Japan, Korea, Lithuania, Norway, Poland, Romania, Russia, Spain, Sweden, Switzerland, Turkey, Ukraine, UK (Yu et al., 2012).

\section{Mesoleptus incessor (Haliday, 1838)}

Material examined: Isfahan province, Khansar (33¹3'14"N 50¹8'54"E), 20', April 2005.

Distribution outside Iran: Austria, Azerbaijan, Belgium, Bulgaria, Cyprus, Czech Republic, Denmark, Estonia, Finland, France, Germany, Greece, Hungary, Ireland, Italy, Latvia, Moldova, the Netherlands, Norway, Poland, Romania, Russia, Spain, Sweden, Switzerland, Turkey, Ukraine, UK (Yu et al., 2012).

\section{Genus Synechocryptus Schmiedeknecht, 1904}

\section{Synechocryptus persicator Aubert, 1986}

Material examined: Isfahan province, Shahreza $\left(32^{\circ} 00^{\prime} \mathrm{N} 51^{\circ} 52^{\prime} \mathrm{E}\right), 1$, , September 2009.

Distribution outside Iran: Armenia, Turkey (Yu et al., 2012).

\section{Subfamily Ichneumoninae \\ Genus Barichneumon Thomson, 1893}

\section{Barichneumon albicaudatus (Fonscolombe, 1847)}

Material examined: Isfahan province, Nain $\left(32^{\circ} 51^{\prime} 36^{\prime \prime} \mathrm{N} 53^{\circ} 05^{\prime} 15^{\prime \prime E}\right)$, 1 \%, July 2008.

Distribution outside Iran: Austria, Azerbaijan, Belgium, Bulgaria, Croatia, France, Germany, Hungary, Italy, Kazakhstan, Lithuania, Macedonia, Poland, Romania, Russia, Spain, Turkey, UK, former Yugoslavia (Yu et al., 2012).

\section{Genus Coelichneumon Thomson, 1893}

\section{Coelichneumon dorsosignatus (Berthoumieu and Eversmann, 1894)}

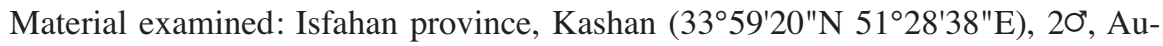
gust 2007.

Distribution outside Iran: Algeria, Austria, Azerbaijan, Germany, Kazakhstan, Poland, Romania, Russia, Spain, Turkey (Yu et al., 2012). 


\section{Genus Ctenichneumon Thomson, 1894}

\section{Ctenichneumon repentinus (Gravenhorst, 1820)}

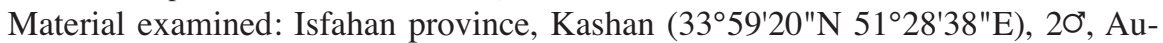
gust 2007.

Distribution outside Iran: Austria, Azerbaijan, Belarus, Belgium, Bulgaria, Croatia, former Czechoslovakia, Finland, France, Germany, Greece, Hungary, Italy, Italy-Sicily, the Netherlands, Norway, Poland, Romania, Russia, Spain, Turkey, Ukraine, UK (Yu et al., 2012).

\section{Genus Hepiopelmus Wesmael, 1845}

\section{Hepiopelmus melanogaster (Gmelin, 1790)}

Material examined: Isfahan province, Khomeyni-Shahr (3242'01"N 5131'16"E), 10 , October 2007. New record for the fauna of Iran.

Distribution outside Iran: Austria, Belarus, Belgium, Bosnia Hercegovina, Bulgaria, former Czechoslovakia, Finland, France, Germany, Hungary, Ireland, Italy, Japan, Latvia, Lithuania, the Netherlands, Norway, Poland, Romania, Russia, Spain, Sweden, Switzerland, Turkey, UK, former Yugoslavia (Yu et al., 2012).

\section{Genus Ichneumon Linneaus, 1758}

\section{Ichneumon coniger Tischbein, 1876}

Material examined: Isfahan province, Semirom (31 $\left.244^{\prime} 51^{\prime \prime} \mathrm{N} 51^{\circ} 34^{\prime} 10^{\prime \prime E}\right), 1$, August 2008.

Distribution outside Iran: Austria, Bulgaria, Croatia, France, Germany, Hungary, Poland, Romania, Serbia, Spain, Sweden, Turkey, Ukraine, former Yugoslavia (Yu et al., 2012).

\section{Ichneumon lautatorius Desvignes, 1856}

Material examined: Isfahan province, Isfahan (1592 m), 1Q, July 2006. Isfahan

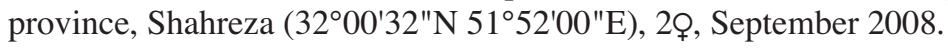

Distribution outside Iran: Austria, Belgium, Bulgaria, China, France, Germany, Hungary, Italy, the Netherlands, Poland, Romania, Spain, Turkey, UK (Yu et al., 2012).

\section{Ichneumon ostentator Heinrich, 1978}

Material examined: Isfahan province, Golpaygan $\left(33^{\circ} 27^{\prime} 13^{\prime \prime} \mathrm{N} 50^{\circ} 17^{\prime} 18^{\prime \prime} \mathrm{E}\right), 10^{\prime \prime}$, 1Q, August 2008.

Distribution outside Iran: Greece, Spain, Turkey, UK, former Yugoslavia (Yu et al., 2012). 


\section{Subfamily Ophioninae \\ Genus Enicospilus Stephens, 1835}

\section{Enicospilus ramidulus (Linnaeus, 1758)}

Material examined: Isfahan province, Golpaygan $\left(33^{\circ} 27^{\prime} 13^{\prime \prime} \mathrm{N} 50^{\circ} 17^{\prime} 18^{\prime \prime} \mathrm{E}\right), 1$, September 2005. Isfahan province, Fereydun-Shahr (3256'28"N 5007'16"E), 1o, 10", June 2006.

Distribution outside Iran: Afghanistan, Algeria, Austria, Azerbaijan, Belarus, Belgium, Bulgaria, Canary Islands, China, Croatia, Czech Republic, Denmark, Egypt, Ethiopia, Finland, France, Germany, Hungary, Ireland, Israel, Italy, Japan, Kazakhstan, Korea, Latvia, Macedonia, Moldova, the Netherlands, Norway, Poland, Romania, Russia, Spain, Sweden, Switzerland, Turkey, Ukraine, UK, former Yugoslavia (Yu et al., 2012).

\section{Genus Ophion Fabricius, 1798}

\section{Ophion luteus (Linnaeus, 1758)}

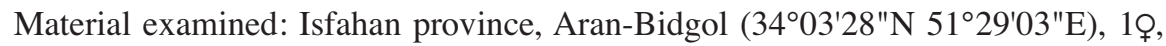
April 2012.

Distribution outside Iran: Algeria, Argentina, Australia, Austria, Azerbaijan, Belarus, Belgium, Bulgaria, Canada, Canary Islands, Chile, China, Croatia, Cyprus, Czech Republic, Estonia, Finland, France, Germany, Greece, Greenland, Hungary, Iceland, India, Ireland, Italy, Italy-Sardinia, Italy-Sicily, Japan, Korea, Latvia, Lebanon, Lithuania, Moldova, Mongolia, Morocco, the Netherlands, Norway, Poland, Portugal, Romania, Russia, Spain, Sweden, Switzerland, Syria, Turkey, USA, Ukraine, UK, former Yugoslavia (Yu et al., 2012).

\section{Subfamily Pimplinae \\ Genus Apechthis Förster, 1869}

Apechthis quadridentata (Thomson 1877)

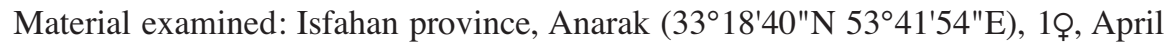
2006. New record for the fauna of Iran.

Distribution outside Iran: Albania, Armenia, Austria, Azerbaijan, Belarus, Belgium, Bulgaria, China, Croatia, Czech Republic, Finland, France, Georgia, Germany, Greece, Hungary, Ireland, Italy, Japan, Kazakhstan, Lithuania, Macedonia, Moldova, Mongolia, the Netherlands, Norway, Poland, Romania, Russia, Serbia and Montenegro, Slovakia, Spain, Sweden, Switzerland, Turkey, Ukraine, UK, former Yugoslavia (Yu et al., 2012).

\section{Genus Pimpla Fabricius, 1804}

\section{Pimpla insignatoria (Gravenhorst, 1807)}

Material examined: Isfahan province, Shahreza ( $\left.32^{\circ} 00^{\prime} 32^{\prime \prime N} 51^{\circ} 52^{\prime} 00^{\prime \prime E}\right), 10^{\prime \prime}$, September 2008 . 
Distribution outside Iran: Armenia, Austria, Azerbaijan, Belarus, Bulgaria, Croatia, Czech Republic, Denmark, France, Georgia, Germany, Hungary, Macedonia, the Netherlands, Poland, Romania, Russia, Turkey, Ukraine, UK, former Yugoslavia (Yu et al., 2012).

\title{
Genus Tromatobia Förster, 1869
}

\section{Tromatobia ovivora (Boheman, 1821)}

Material examined: Isfahan province, Fereydun-Shahr (32॰56'28"N 5007'16"E), 1 , June 2006.

Distribution outside Iran: Austria, Azerbaijan, Belarus, Belgium, Bulgaria, Canada, Croatia, Czech Republic, Finland, France, Germany, Hungary, Ireland, Italy, Italy-Sicily, Japan, Kazakhstan, Kyrgyzstan, Latvia, Lithuania, Macedonia, Mexico, Mongolia, the Netherlands, Norway, Poland, Romania, Russia, Serbia and Montenegro, Spain, Sweden, Switzerland, Turkey, USA, UK, former Yugoslavia (Yu et al., 2012).

\section{Subfamily Tryphoninae Genus Kristotomus Mason, 1962}

\author{
Kristotomus laetus (Gravenhorst, 1829) \\ Material examined: Isfahan province, Khansar (3313'14"N 50¹8'54"E), 2Q, April \\ 2005.
}

Distribution outside Iran: Armenia, Austria, Belgium, Bulgaria, former Czechoslovakia, Denmark, Finland, France, Georgia, Germany, Greece, Hungary, Ireland, Italy, Latvia, Lithuania, Mongolia, the Netherlands, Norway, Poland, Romania, Russia, Spain, Sweden, Switzerland, Turkey, Ukraine, UK (Yu et al., 2012).

\section{Genus Phytodietus Agassiz, 1846}

\section{Phytodietus polyzonias (Forster, 1771)}

Material examined: Isfahan province, Semirom ( $\left.31^{\circ} 24^{\prime} 51^{\prime \prime N} 51^{\circ} 34^{\prime} 10^{\prime \prime E}\right), 1$, $\mathrm{Au}-$ gust 2008 .

Distribution outside Iran: Armenia, Austria, Azerbaijan, Belarus, Belgium, Bulgaria, China, Czech Republic, Denmark, Finland, France, Georgia, Germany, Hungary, Ireland, Italy, Kazakhstan, Korea, Latvia, Lithuania, Moldova, the Netherlands, Norway, Poland, Romania, Russia, Serbia and Montenegro, Slovakia, Spain, Sweden, Switzerland, Turkey, Ukraine, UK (Yu et al., 2012). 


\section{Discussion}

In this research 28 species from 24 genera of the family Ichneumonidae were identified from different regions of Isfahan province. The results of this survey indicate the existence of a diverse range of the ichneumonids in the mentioned region. Several of the collected Ichneumonidae are still waiting for subsequent determinations. The Ichneumonidae is the largest family within Hymenoptera, and one of the largest among insects (Humala and Reshchikov, 2014). Although in total more than 500 species of Ichneumonidae have been listed from Iran so far (Barahoei et al., 2012), the published data from the adjacent countries and also presence of various geographical regions and climates in Iran suggest that the Iranian list is still very incomplete. The faunistic works on Iranian Ichneumonidae are continued in different areas and so these surveys will result to new data toward the completing of Iranian Ichneumonidae list.

\section{Acknowledgements}

The authors are grateful to R. Jussila (Finland) and M. Schwarz (Austria) for their cooperation in this research. The research was supported by Islamic Azad University (Yadegar - e-Imam Khomeini (RAH) Branch), Iran and Cairo University, Egypt.

\section{Literature}

Barahoei, H., Rakhshani, E. and Riedel, M. (2012): A checklist of Ichneumonidae (Hymenoptera: Ichneumonoidea) from Iran. Iranian J. Animal Biosystematics (IJAB) 8, 83-132.

Barahoei, H., Rakhshani, E., Fathabadi, K. and Moradpour, H. (2014a): A survey on the fauna of Ichneumonidae (Hymenoptera) of Khorasan-e-Razavi province. Iranian J. Animal Biosystematics (IJAB) 10, 145-160.

Barahoei, H., Nader, E. and Rakhshani, E. (2014b): Cryptinae (Hymenoptera: Ichneumonidae) of Isfahan province, central Iran. Turkish J. Zoology 39, 279-284.

Barahoei, H., Nader, E. and Rakhshani, E. (2015): A survey on Ichneumonidae of Isfahan province, central Iran. J. Crop Protection 4, 157-166.

Çoruh, S., Kolarov, J. and Çoruh, I. (2014): Probles microcephalus (Gravenhorst, 1829) a new record for the Turkish fauna (Hymenoptera: Ichneumonidae: Tersilochinae). Munis Entomology and Zoology 9, 451-456.

Ghahari, H. and Jussila, R. (2014a): A faunistic study on the Ichneumonidae (Hymenoptera: Ichneumonoidea) from the west of Iran. Linzer biologische Beiträge 46/2, 1373-1377.

Ghahari, H. and Jussila, R. (2014b): A study on the subfamily Ichneumoninae (Hymenoptera: Ichneumonidae) from Khorasan province, Iran. Linzer biologische Beiträge 46/2, 1367-1371.

Ghahari, H. and Jussila, R. (2015): Faunistic notes on the Ichneumonid wasps (Hymenoptera: Ichneumonidae) in alfalfa fields in some regions of Iran. Entomofauna 36, 185-192.

Humala, A. E. and Reshchikov, A. (2014): Ichneumonidae (Hymenoptera) species new to the fauna of Norway. Biodiversity Data Journal 2, 1-26.

Townes, H. (1969): The genera of Ichneumonidae, Part 1. Memoirs of American Entomological Institute 11, $1-300$.

Yu, D. S., Achterberg, K. and Horstmann, K. (1997): A catalogue of world Ichneumonidae (Hymenoptera). Memoirs of the American Entomological Institute 58, $1558 \mathrm{p}$.

Yu, D. S., van Achterberg, C. and Horstmann, K. (2012): Taxapad 2012, Ichneumonoidea 2011. Database on flash-drive, www.taxapad.com, Ottawa, Ontario, Canada. 